\title{
Heart Rate Score
}

National Cancer Institute

\section{Source}

National Cancer Institute. Heart Rate Score. NCI Thesaurus. Code C81309.

A component of the Apgar score, it is the numerical value assigned to an assessment of the circulatory status of a neonate. $0=$ no pulse; 1 = rate less than 100 beats per minute; $2=$ rate greater than 100 beats per minute. 